\title{
Fundamentals of the Islamic Medical Philosophy
}

\author{
Shogar, Ibrahim A. \\ Kulliyyah of Science, International Islamic University Malaysia Kuantan
}

\begin{abstract}
The purpose of this paper is to advocate for a holistic medical philosophy that is capable of accommodating various models of medical explanations. Such a comprehensive and integrated approach of medical philosophy should be capable of addressing, the ethical, epistemological and metaphysical aspects of medical research and practice. The basic presupposition of the paper is that the Islamic principles on health promotion can play an important role in developing such a holistic medical philosophy. Adopting the analytic method, the paper investigates the three major frameworks of explanation that dominated the history of medical philosophy: (a) the humoral model that is based on balance of the humors; (b) the biocentric model which is based on causal explanation and defines disease in descriptive biological terms; and (c) the postmodern or meta-biocentric model that concerned mainly with the medical philosophy and aims at promoting the holistic approach. This paper, also, investigates the three basic forms of medical explanations which are determined by the above domains, i.e. the descriptive, the normative, and the integrated models. The paper traces the impact of explanation methods on good medical practices and explores the Islamic medical philosophy and its potential role in promoting the holistic approach. Along the way, the paper discusses the ideal relationship between physician and patient according to the Islamic perspective.
\end{abstract}

KEYWORDS: medical philosophy, health promotion, socio-cultural aspects, causal explanation, normative approach.

\section{INTRODUCTION}

History of medical practices is traced back to the history of mankind on the planet earth. Promotion of health, as a major means to perpetuating 'life' against 'death' which comes through 'disease' and 'sickness', might be regarded as an innate character of human being. Mankind across the history has been concerned with understanding the natural forces and the risk factors that affect his health and life. Human community, thus, has made considerable efforts to achieve this noble goal, based on distinctive approaches. However, to deal with health matters and disease problems in a systematic manner, man needs knowledge and experience; and before that he needs to answer many critical questions on various phenomena, such as what is disease, what causes sickness, and why we must die? What are the most effective methods to prevent disease and promote health? The proper answer to such fundamental questions necessitates not only the medical, but also the social, economical and cultural aspects of human life. The theoretical study of such problems of health and disease constitutes the basic subject matter and the main objective of medical philosophy.

Corresponding author:

Shogar, Ibrahim A.

Kulliyyah of Science,

International Islamic University Malaysia

Jalan Sultan Ahmad Shah,

25200 Kuantan,

Pahang, Malaysia

Email: shogar@iium.edu.my
There are various forms of medical philosophies, but the three major frameworks of explanation that dominated the history of medical practice are: the humoral, biomedical, and the post-biomedical models. The major differences between the three philosophies are defined by both their framework of explanation, i.e. conceptualization of the medical phenomena, as well as by their methods of application and practices. This article presents, briefly, the major role that can be played by the Islamic medical philosophy in bridging the gap between these approaches. It introduces principles of health promotion in Islamic account as foundations for promoting the integrated model.

\section{FUNDAMENTALS OF THE MEDICAL PHILOSOPHY}

The medical philosophy deals with different issues of 'health' and 'disease' at various levels. This includes the conceptual analysis of key terms; the constructive review of fundamentals of medical professions; the critical examination of therapeutic approaches; addressing the ethical and the metaphysical questions of medicine; developing the alternative methods for efficient, effective, and the peaceful medical practice. From a pragmatic perspective, the medical philosophy distinguishes between two distinctive levels of medical intervention:

i. The preventive level that is concerned with matters of public healthcare that aims at promoting health through disease prevention (promoting a healthy life). This level is regarded as a strategic approach and considered as a basic medical method in the 
developed communities. It addresses several issues, related to the preventive measures, such as self-awareness, natural ecology, and socio-cultural aspects of human life. Social class matters, economics, and the equal access to medical and health services care are arise at this level. ${ }^{1}$

ii. The clinical intervention level that deals with individual patients based on the relevant therapeutic methods. The two major steps of clinical intervention level are recognition of the disease or illness precisely (diagnosis), and the effective method of treatment. The importance of both the clinical and patient explanatory models appears at this level. The major problems of medical philosophy, including the ethical and epistemological questions, also, arise at this level. Arthur Kleinman, Leon Eisenberg, et al. ${ }^{1,2}$, note that it is crucial to recognize that patient-doctor interactions are transactions between explanatory models; transactions often involving major discrepancies in cognitive content as well as therapeutic values, expectations, and goals. They further emphasize that a patient's explanatory model and view of clinical reality can be quite discordant with the professional medical model, producing misunderstanding and problems in clinical management. ${ }^{2}$

In fact, the two levels of medical engagement are interconnected, in the sense that promotion of health at public level, a 'healthy life', promotes healthcare at an individual level; and good clinical practices at an individual level on the other hand are necessary to promote healthcare at collective level.

Philosophy of medicine deals with all these matters and other theoretical issues of medical research and practice. It investigates the fundamental questions on nature of the medical phenomena and conducts the conceptual analysis, aiming at answering the epistemological, ethical, and ontological questions of medicine and healthcare professions. The fundamental characteristics of medical philosophy are analytic and theoretical in nature. The distinctive modes of medical philosophy are determined by their rational framework of explanation. The continuous attempts to conceptualize the phenomena of sickness and disease, along the history of medicine, have formed three basic modes of explanation to the nature of medical phenomena: (a) classical medicine, (b) biobased medicine, and (c) social medicine. The three models are aiming at investigating the same phenomena but based on different frameworks. Any attempt to defining the key concepts of medical science, such as disease, sickness, and healing, must be guided by one of these models. These approaches, on the other hand, explain various forms of explanation, which can be divided mainly into: (i) normative, (ii) descriptive, and (iii) integrated $^{(\mathrm{i}) \text {. }}$

\section{The Normative Approach}

The 'normative' approach explains the entire phenomena and medical concepts, such as health, disease, and sickness, in the context of cultural norms and moral judgements. It focuses on the nosological level of 'disease', rather than the pathophysiological or psychopathological aspects of disease mechanisms which is explained by causal relationship ${ }^{3}$. The society judgment is the dominant factor in this case. Emphasizing the social aspects of medicine, Ian Gregory explains that, "While professionals have a major voice in influencing the judgment of society, it is the collective judgment of the larger social group that determines whether its members are to be viewed as sick or criminal, eccentric or immoral"4,(ii)

The historical background of the normative explanation might be traced back to the ancient Greek medical philosophy, known as the 'humoral model', which dominated the medical thought up to the Middle Ages. It was based on teleological framework of explanation which understand the world based on functions and definite purposes. The ancient Greek philosophers thought that good health is due to a perfect balance of the four humors; blood, phlegm, yellow bile, and black bile. A poor health, on the other hand, is due to an improper balance of the four humors. Accordingly, cure means to restore balance of the body through various means and different therapeutic methods. Humoral model considers both the spiritual and material dimensions of human body. The philosophical foundations of Greek medicine, however, were derived from different sources, such as the humors, gender, social class, geographic location, diet, and mindset. For example, they believed that gender plays an important role in medical theory because some diseases of women and treatments are different from that of men. Hippocrates and Galen were the founding fathers of this model who had contributed in the development of Greek medical philosophy which dominated until the Middle Ages. They also contributed in the development of medical ethics and exposed physicians to the Hippocratic Oath which is valid until today ${ }^{5}$

\section{Descriptive Method}

Modern medicine is part of the scientific revolution and its emphasis on observation, experiment, and rationality. As rightly observed by many authors, when the scientific revolution started, the change was not only epistemological but also ontological as well. Nature and human bodies now become regarded as purpose-less mechanical units. Earlier, the human body was understood in analogy with the appearances of animals and plants. At the outset of the scientific revolution, explanations by final causes were banned from physics and biology. The clock with its clockwork became the exemplar and model for how to understand and explain changes. Behind the visible minute and hour hands and their 
movements there is the invisible clockwork mechanism that makes the hands move ${ }^{5}$.

The 'descriptive'(iii) mode of explanation, also known as naturalist approach, is the foundation of modern thought. The historical background of this model is found in the deterministic worldview which formulated principles of modern philosophy of science. The basic assumption and the general presumption of this model is that there are biological features which are common to different types of disease, and it is a scientist or a medical philosopher's job to determine what they consist of . ${ }^{3}$

The most influential figures in the development of the descriptive approach in medical philosophy are Rene Descartes (d. 1650) and Immanuel Kant (d. 1804). The former extended the mechanical method of physical sciences to human sciences, especially to the medical science, reducing human body into a mechanical agent that functions in systematic orders based on causal relationships. ${ }^{\text {(iv) }}$ While the later, Kant, developed metaphysics of moral philosophy, emphasizing on human freedom, extended the ethical concerns to medical research. Thus, this model also known as 'Cartesian' or 'Kantian', due to contribution of the two philosophers in the development of epistemological and ethical principles of modern medical philosophy.

Cartesian reductionism views the body as a machine that functions in a specific manners and medical professionals as technicians whose job is to repair that machine. ${ }^{6}$ The reductionist approach is the dominant method in present-day medical science, which could be named as 'the clinical medical paradigm'.5 Major characteristic of this model is that it disregards the metaphysical parts of human body, such as spirit and soul, thus it deals solely with the physical part of human body which regarded as a 'machine' that functions in accordance to systems measurable by empirical means. Beside problems of biomedical ethics and patient-physician relationship, the epistemological questions of the descriptive approach with regard to the medical research are centered around the randomized controlled trial. ${ }^{5}$

\section{The Integrated Approach}

The integrated approach aims at developing a holistic method of explanation on medical phenomena that takes into consideration all questions of medical enterprise, either related to nature of human body, definition of disease and illness and the therapeutic method, or related to patient-doctor relationship. This approach might be regarded as 'meta-biobased' approach, because it is a result of critical review of principles of modern evidence-based medical philosophy. ${ }^{(v)}$ It attempts to overcome the shortcomings of the other approaches, by integrating both the classical and modern, or between the value-based and evidence-based philosophies into a single method of explanation. In all cases, conceptualization of 'disease' is the central issue.

The current literature on medical philosophy shows that, the conceptual analysis is dominant, beside the biomedical ethics, with less focus on other domains of medical philosophy such as epistemological and ontological problems. According to Maël Lemoine, most of medical philosophers who attempted to capture the meaning of 'disease' in medical science have tried to draw it from "disease judgments" approach rather than from what he called "disease explanations". According to him, a "disease judgment", is a judgment that some condition is or is not a disease, ideally grounded on some explicit, uncontroversial nosography. A disease explanation, on the other hand, could be defined as a causal model of what is happening in a specific pathological condition, and it belongs to pathophysiology. ${ }^{3}$

The basic characteristic of integrated model is that it deals with all problems of medical philosophy in a holistic manner. It does not focus only on clinical ethics but also the other domains of medical philosophy. It aims to develop an alternative approach that, instead of trying to find the correct definitions of key concepts such 'health' and 'disease', it focuses more on central issues of medical philosophy, such as description of physiological or psychological states; and normative claims, claims about what states we value or disvalue. Some authors suggest that such distinction would avoid the problems facing the major approaches to defining 'health' and 'disease', and it more clearly captures what matters in medical discussions. Some medical philosophers have held the view that the integrated approach would ultimately lead to a general theory of disease linked with the other general theories on living beings, among them, systems biology and network medicine.

\section{FUNDAMENTALS OF ISLAMIC THE MEDICAL PHILOSOPHY}

Conceptualization of 'health' and 'disease', must be based on a rational framework of understanding of a medical philosophy that is determined by a specific mode of explanation. This framework of explanation can be named as 'model', paradigm, or worldview. Fundamentals of health promotion in Islamic account are determined by the principle objectives of the Islamic worldview that aim at preservation of human life, mind, and progeny. Islam, a holistic view of life, provides principles of medical philosophy rather than detailed knowledge of medical practices. In fact, all the central issues of medical philosophy, such as the key medical concepts, issues of social medicine, and the metaphysical foundations of interpreting are found in the Islamic medical philosophy. This is in addition to the moral philosophy that involves in determining the crucial role of human values in medical research and critical examination of ethical questions posted by modern advancements in science and technology, 
especially biomedical ethics.

Ingvar Johansson and Niels Lynøe rightfully observe that during the middle ages when the Islamic culture was the most advanced scientificphilosophical culture in the world, prominent Muslim philosophers such as Ibn Sina (Avicenna) and Ibn Rushd (Averroes) made substantial contributions to both philosophy and medicine. This was due to the fact that the holy Qur 'an was their motivational force. Thus, revival of such an integrated culture of medicine and philosophy is essential in modern Muslim intellectual's society. ${ }^{5}$

The holy Qur'an, as the final mode of Divine revelation, addresses the fundamental questions of human thought and exposes mankind to all that is essential for a meaningful life. This includes the relevant answers to the metaphysical questions of human thought that are necessary to establish the worldview; addressing the epistemological problems of knowledge and truth; and providing principles of moral values as well as the detailed knowledge of the right conduct. Health matters in Islamic account are, therefore, understood and practiced in social and moral contexts. The holy Qur 'an has used the term 'disease' (مرض) more than ten times for both physical and moral 'illness', but majority of these cases were about moral 'sickness'. Thus, to enjoy a healthy and meaningful life, in Islamic account, one must be free not only from physical diseases and illness, but more importantly, from moral diseases which the holy Qur' an named as 'disease in the heart'. Accordingly, a sound worldview is essential to establish effective moral values which guide to healthy lifestyles. Therefore, the principles of Islamic medical philosophy are firmly connected with the Islamic worldview. These principles are aiming at promoting health through solving the metaphysical, epistemological and ethical problems in medical research and practice.

Conceptualization of Heath and Disease in the Islamic Medical Philosophy

Health, disease and sickness are complex medical concepts that indispensably related to human life. The analytical study of such concepts is a subject matter of medical philosophy which aims at perfection of healthcare methods. The critical problem of such attempt however arises from the fact that these concepts are elusive. The World Health Organization (WHO) has defined health as "a state of complete physical, social and mental wellbeing, and not merely the absence of disease or infirmity". ${ }^{7}$ This attempt, in fact, describes what 'should be' rather than 'what is'.

What 'should be', however, cannot be without consideration of the ethical aspects of a healthy lifestyle. Conceptualization of health and disease in the Islamic perspective is, therefore, connected with consideration of both physical, spiritual and psychological components of human being. The process of conceptualizing such concepts is, accordingly, guided by the fundamental objectives of the Islamic medical philosophy which focused on preservation of human life. The major means to achieve this noble goal, in Islamic account, include establishing a firm culture of healthy lifestyle, prevention of diseases, medication, and finally the positive attitude for better live in a life to come. Thus, conceptualization of 'health' and 'disease' must be in the rational framework of the Islamic worldview which connects this temporal life firmly with the absolute life of the hereafter. The concept of 'health' in the Islamic medical philosophy is determined not only by external factors of physical well-being but also, and more importantly, by internal factors of emotional self-management. The internal factors include positive attitudes, good intentions, and optimism for better life in hereafter. That is why the holy Qur' an persistently remind us about the holistic meaning of 'health' by shifting constantly our attention to "disease in heart". The modern research on "placebo effect" has confirmed the later type of disease.

The following are the major characteristics of the Islamic medical philosophy:

1. Guided by fundamentals of the Islamic worldview, i.e. unity of God as central for explanation;

2. Preventive rather than curative;

3. Holistic rather than reductionist, both in sense of:

a.Conceptualization of the medical phenomena;

b.Method of treatment.

4. Patient-centered rather than doctorcentered;

5. Bio-spiritual based rather than causal explanation.

Based on these characteristics, the Islamic medical philosophy can serve medicine as follows:

$\Rightarrow \quad \begin{aligned} & \text { Providing metaphysical foundations of } \\ & \text { medical science; }\end{aligned}$
$\Rightarrow \quad \begin{aligned} & \text { Answering epistemological questions of } \\ & \text { medical \& healthcare professions; }\end{aligned}$
$\Rightarrow \quad \begin{aligned} & \text { Solving ethical problems of medical research } \\ & \text { \& practice; }\end{aligned}$
$\Rightarrow \quad \begin{aligned} & \text { Providing medical concepts and } \\ & \text { terminologies; }\end{aligned}$
$\Rightarrow \quad \begin{aligned} & \text { Promoting healthy lifestyles through both the } \\ & \text { internal power of morality and external power } \\ & \text { of law. }\end{aligned}$

The Metaphysical Foundations of the Islamic Medical Philosophy

The metaphysical principles of medical philosophy are that which concerned with the nature of humanity, the nature of sickness and the meaning of disease. Scientists believe that biological processes are very complex, but human phenomenon is even more complex because it is imparted by material, 
spiritual, and rational factors. Some of these parts are physical while others are metaphysical. Due to its complexity, understanding the nature of humanity has been a challenging factor for both philosophers and scientists. Views on this matter are always conflicting and controversial. The holy Qur'an has solved this problem by attributing knowledge of all metaphysical parts of humanity, such as spirit and soul, to the Creator. We should follow the Qur'ānic directives, saying: (Who created me shall give me the guidance, He who gives me food and drink, and when I get sick He who gives me cure). ${ }^{\text {(vi) }}$

It is very rational that who gives life to a body and affects that body by disease can give cure as well. But human needs to investigate to find where the cure is hidden within the diverse creatures of the natural phenomena. There are two methods to promote health in the Islamic medical philosophy, first, adhering to divine guidance with regard to metaphysical parts of humanity; and second, investigation of the natural phenomenon to uncover the cure from where it hidden, because the Divine revelation has informed that: (for every disease, there is a cure).

\section{The Epistemological Foundations of Health Promotion}

The epistemological principles of health promotion aim at promoting knowledge and awareness at both public healthcare and clinical intervention levels (protective \& curative). Accordingly, the major problems of medical epistemology are (i) claims of awareness on illness mechanism, and how can it be verified by conventional and non-conventional medical approaches; and (ii) claims of effectiveness with respect to a specified symptom or illness that can be verified only for a particular approach employed in a specified system of medicine. Thus, in many cases selecting the appropriate clinical method when addressing a specified illness requires judgments other than empirical detection or verification of a putative mechanism of action. ${ }^{8}$ These epistemological principles are mainly related to material aspects of human body, where cure of every disease is available, but needs investigation to uncover its nature and its descriptive feature. With regard to the epistemological domain of health promotion; the holy Qur an provides hints and guidelines which lead mankind to develop a firm culture of a healthy lifestyle. It forbids all harmful substances and destructive acts, because a healthy lifestyle is a major method to maintain human being alive and in good condition to uphold the great mission that Islam shoulders on him.

The epistemological principles of the Islamic medical philosophy are concerned with both knowwhat and know-how questions in medical science. The purpose of basic medical research is to obtain know-that. But the overarching knowledge purpose of the whole healthcare system, which much of the medical research is a part of, is to develop know- how. It embodies knowledge about how to prevent diseases and illnesses, how to diagnose diseases and illnesses, how to treat diseases and illnesses, how to alleviate pain, and how to comfort a patient. ${ }^{5}$

Prof Rashid Bhikha in his paper "The Islamic Medicine Revisit" suggests that Islamic Medicine should adhere to the theoretical and philosophical principles of the temperamental and humoral theories from the Qur 'an and Sunnah, and should be practiced in accordance with the concepts, values, and procedures of Islamic law. He holds that the development of Islamic medicine in the context of Greek, Chinese and Ayurvedic medicine has led to several common concepts, such as inner healing and internal harmony, the adoption of a holistic approach to healing, and the role of spiritual influence. However, Bhikha observes that there are significant differences between Islamic medical philosophy and other, such as the difference in the worldview and the concept of creation. ${ }^{9}$

\section{The Ethical Foundations of Health Promotion}

Moral integrity and ethical principles play an important role in both the Islamic medical philosophy as well as the conventional approach. These principles aim at developing ethical norms to guide medical research and practice. Many authors have emphasized that ethical questions of medicine should not be narrowly restricted to the usual topics of bioethics, ranging from abortion, assisted reproduction, and euthanasia, to genetic engineering, cloning, organ sales, and the allocation of medical resources; but must be extended to address all medical problems. They note that all these issues are shaped by foundational views regarding the nature of the physician-patient relationship and the goals of medicine, all of which are the proper focus of the philosophy of medicine. Besides the biomedical ethics, there are principles of clinical research ethics which includes autonomy, beneficence, non-maleficence, justice, solidarity, and vulnerability. ${ }^{10}$

The ethical principles also address the urgent need to develop new patient-physician relationship instead of the current economic derived relationship. For this matter, Pellegrino and Thomasma propose an alternative philosophy of medicine that defines medicine as "a relation of mutual consent to effect individualized well-being by working in, with, and through the body". ${ }^{11,1}$ They propose that that the center of medicine is relationship, and the purpose of the relationship is healing. ${ }^{13,14}$ This is not to deny the importance of technical competence gained from the reductionist scientific enterprise. ${ }^{6}$ (vii) $^{2}$

Islamic philosophy of science promotes all the above fields of medical ethics by codification of the ethical norms which determine the ideal relationship between patient and physician based on principles objectives Shari'ah. It also provides guidelines to promote ethics of clinical research as well as the all forms of biomedical ethics. 


\section{CONCLUDING REMARKS}

Various problematic factors are contributing to emphasize the dire need for a holistic model of medical philosophy that integrates between the descriptive and the normative methods of explanation. These factors include (i) the necessity for standard definition of the key medical terminologies, such as health, disease, and sickness; (ii) developing a holistic perspective that takes in account the medical ecology and socio-cultural aspects of medicine; (iii) the urgent need for reshaping the dominating economic driven model of doctor - patient relationship that looks at patients merely as a source of income; and (iv) the need for an urgent answer to the critical ethical questions posted by medical research and practice, specially biomedical research.

According to Robert $M$. Veatch, the idea that considers the twentieth century as the high point in progress of modern medicine has passed. New medical philosophes have developed to challenge philosophies of modernity medicine, a period that encompasses the epitome of our understanding of how medicine came to be viewed as clinical science. According to this view, physicians are expected to be able to rely on modern science to determine what is best for patients and to prescribe for them 'treatment choice' or 'best medical practice' that has the highest probability of benefiting the patient by restoring or preserving health. This view of scientifically based medicine has already become the victim of a number of philosophical challenges that changed our understanding of the practice so radically. The era of modern medicine (bio-based) is rapidly coming to an end. It is being replaced by what can be called as post-modern or contemporary medicine. ${ }^{11}$

The Islamic medical philosophy that integrates the physical, spiritual, and mental aspects in its philosophy is expected to play an important role in bridging the gap between the above two approaches, i.e. modernism post-modern medicines, which represented by descriptive and normative approaches. The distinctive principles of health promotion in the Islamic medical philosophy are found at various levels: (i) the ontological principles, which aim at establishing the metaphysical foundations of 'health' and 'disease', such as faith in God Who creates both the disease and the cure, therefore $\mathrm{He}$ is the final cause of healing; (ii) the declarative (normative) principles, which aim at providing the general guidelines to achieve the goal of a healthy life, such as creating self-awareness, forbidding all harmful substances / items, destructive habits, and unhealthy lifestyles; (iii) epistemological and methodological principles, which concerned with professionalism and knowledge of therapeutic methods, such as integration between normative and descriptive approaches; (iv) the ethical principles that aim at developing the moral values to maintain integrity of medical research and practice.
The Islamic medical philosophy, unlike the conventional, is more concerned with issues of social medicine that emphasize the protective measures of health promotion. In the introductory remarks for their eminent work "Culture, Illness, and Care". Arthur Kleinman, Leon Eisenberg, et al. 2,1 , promote the holistic perspective as following: "Major health care problems such as patient dissatisfaction, inequity of access to care, and spiraling costs, no longer seem amenable to traditional biomedical solutions. Concepts derived from anthropologic and cross-cultural research may provide an alternative framework for identifying issues that require resolution. A limited set of such concepts is described and illustrated, including a fundamental distinction between disease and illness, and the notion of the cultural construction of clinical reality. These social science concepts can be developed into clinical strategies with direct application in practice and teaching. One such strategy is outlined as an example of a clinical social science capable of translating concepts from cultural anthropology into clinical language for practical application".

It is the author's aspiration that this paper will generate further discussion on this matter, leading to the development of a holistic and universal healthcare philosophy of medicine.

\section{REFERENCES}

1. Eisenberg L. Does Social Medicine still matter in an era of Molecular Medicine? J Urban Health 1999; 76(2):164-175.

2. Kleinman A, Eisenberg L, Good B. Culture, illness, and care: clinical lessons from anthropologic and cross-cultural research. Ann Intern Med 1978; 88(2):254.

3. Lemoine $M$. Defining disease beyond conceptual analysis: An analysis of conceptual analysis in philosophy of medicine. Theor Med Bioeth 2013; 34(4):30925.

4. Margolis J. The concept of disease. J Med Philos 1976; 1(3):238-255.

5. Johansson I, Lynøe N. Medicine \& Philosophy: A Twenty-First Century Introduction. In Gazelle Books Services: United Kingdom 2008; 173-174.

6. Scott JG et al. Philosophy, ethics, and humanities in medicine: Licensee BioMed Central Ltd 2009;1.

7. World Health Organization WHO, website at http: / /www.who.int/suggestions/faq/en/). Accessed 10 December 2018.

8. Lake J. Philosophical problems in medicine and psychiatry. Integrative Medicine 2007;6 (2): 40 .

9. Bhikha R. The Islamic Medicine revisit. J Islamic Med Assoc. http:// www.tibb.co.za/articles/islamic\% 20medicine\%20revisited.pdf. Accessed 10 December 2018. 
10. Engelhardt HT Jr, Jotterand F. The Philosophy of Medicine Reborn: A Pellegrino Reader: University of Notre Dame Press 2008; p 2.

11. Veatch RM. How philosophy of Medicine have changed Medical Ethics. J Med Philos 2006; 592.

12. Pellegrino ED. Toward a reconstruction of medical morality. Am J Bioeth 2006; 6(2):6571.

13. Pellegrino ED, Thomasma DC. A philosophical basis of Medical Practice: Toward a Philosophy and Ethic of the Healing Professions. New York: Oxford University Press 1981.

14. Pellegrino ED. The Internal Morality of Clinical Medicine: a Paradigm for the Ethics of the Helping and Healing Professions. J Med Philos 2001; 26(6):559-79.

15. Moravcsik J. Ancient and modern conceptions of health and medicine. J Med Philos 1976;1(4): 337-347.

16. Rassool GH. The crescent and Islam: healing, nursing and the spiritual dimension. Some Considerations Towards an Understanding of the Islamic Perspectives on Caring. J Adv Nurs 2000; 32(6):1476-84.

17. DeGolia PA. An Alternative View of Health Promotion and Disease Prevention in Eldercare. In Health Promotion and Disease Prevention: A Handbook for Teachers, Researchers, Health Professionals and Decision Makers. Donev D, Pavlekovic G, and Kragelj LZ (Eds):Hans Jacobs Publishing Company, Germany 2007.

18. Doncho $\mathrm{D}$ et al. Health Promotion and Disease Prevention: A Handbook for Teachers, Researchers, Health Professionals and Decision Makers: Hans Jacobs Publishing Company, Germany.

\section{FOOTNOTES}

(i) Different terminologies are used in the contemporary medical literature to descript these approaches, but their distinctive characteristics are determined by their rational frameworks of explanation, their worldviews, either it is a teleological, deterministic or indeterministic. The normative approach interprets the medical phenomena in the context of teleological worldview which explains world in the terms of its function and purpose of being. The descriptive approach is based on deterministic view of the world and causal interpretation, therefore it depends on empirical evidences. The third approach, guided by principles of social medicine, attempts to develop a holistic method of explanation by integrating the other two approaches, i.e. value-based and evidence-based methods.

(ii) Gregory 1968, p. 32. Cited from Margolis, Joseph (1976). The Concept of Disease (The Journal of Medicine and Philosophy, 1976, vol. 1, no. 3). (iii) The term "descriptive" is used in this context for modern philosophy of science that validates science only based observable evidences and measurable factors. It is an evidence-based approach that might be regarded as antitheses to value-based approach. Domination of descriptive method was a result of modernity, which often referred as a combination of science, rationality, democracy, and industrialization. Study more on this matter: (Scott, John G., et al., 2009).

(iv) Although theory of causation plays a crucial role in modern philology of science as general, but it regarded especially important with philosophy of medicine, because it explain the relationship between disease and sickness from one side and between medicine and cure / healing from the other.

(v) Study more on this view, Veatch, Robert $M$ (2006). How Philosophy of Medicine have Changed Medical Ethics "The journal of Medicine and Philosophy, January 2006", p 592

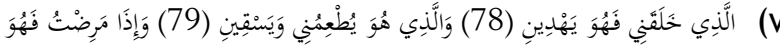

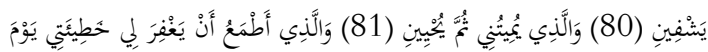

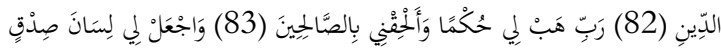

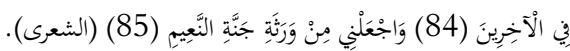

(vii) Cited from Scott, John G., et.al. (2009). Philosophy, Ethics, and Humanities in Medicine (licensee BioMed Central Ltd). P2. 
